1 Fundação Oswaldo Cruz (Fiocruz), Centro de Pesquisas Aggeu Magalhães - Recife (PE), Brasil. rebecca_soaresandrade@ yahoo.com.br

2 Instituto de Medicina Integral Professor Fernando Figueira (Imip) Recife (PE), Brasil.

lua_cad@yahoo.com.br

3 Fundação Oswaldo Cruz (Fiocruz), Centro de Pesquisas Aggeu Magalhães - Recife (PE), Brasil.

freese@cpqam.fiocruz.br

4 Institut de Recherche pour le Développement, Montpellier, Franca. annick.fontbonne@ird.fr

5 Fundação Oswaldo Cruz (Fiocruz), Centro de Pesquisas Aggeu Magalhães - Recife (PE), Brasil. educesse@cpqam.fiocruz.br

\section{Rede de Atenção à Saúde ao portador de Diabetes Mellitus: uma análise da implantação no SUS em Recife (PE)}

\author{
Health Care Network to people with Diabetes Mellitus: an analysis of \\ implementation in the SUS in Recife (PE)
}

Rebecca Soares de Andrade Fonseca dos Santos', Luciana Caroline Albuquerque Bezerra ${ }^{2}$, Eduardo Freese de Carvalho $\mathbf{3}$, Annick Fontbonne $\mathbf{4}^{\mathbf{4}}$ Eduarda Ângela Pessoa Cesse $\mathbf{5}$

RESUMO O artigo avalia a implantação da Rede de Atenção à Saúde aos portadores de Diabetes Mellitus no SUS em Recife, Pernambuco. Trata-se de uma pesquisa avaliativa, do tipo análise de implantação nos níveis da atenção primária e especializada. Os resultados demonstram implantação parcial da Rede em relação às dimensões de estrutura e processo de trabalho. $\mathrm{O}$ contexto político de gestão mostrou-se favorável à implantação da Rede, muito embora ainda existam obstáculos a serem superados.

PALAVRAS-CHAVE Avaliação em saúde; Assistência à saúde; Doenças crônicas; Diabetes Mellitus.

ABSTRACT The article evaluates the implementation of the Health Care Network to diabetic patients in the SUS in the city of Recife, Pernambuco. It is an evaluative research, of the type implantation analysis in the levels of primary and specialized care. The results demonstrate partial implementation of the Network in relation to the dimensions of the structure and work process. The political context of management was favorable to the implementation of the Network, although there are still obstacles to be overcome.

KEYWORDS Health evaluation; Delivery of health care; Chronic disease; Diabetes Mellitus. 


\section{Introdução}

A partir da década de 1990, foram realizadas reformas nos sistemas de saúde de diversos países da América Latina com vistas a ampliar o acesso e qualificar os serviços de saúde. Nesse contexto, no Brasil, as Redes de Atenção à Saúde (RAS) se constituem em uma proposição relativamente recente. A sua implementação pretende adequar e ampliar os serviços prestados em consonância com as necessidades de saúde das populações. Nessa lógica, elas propõem o rompimento da gestão baseada na oferta e investem na integralidade da assistência e longitudinalidade do cuidado. Essa tem sido a forma apontada como mais eficaz para enfrentar o incremento da prevalência das doenças crônicas (SILVA; MAGALHÃES, 2011).

Em 2007, as Doenças Crônicas Não Transmissiveis (DNCT) foram responsáveis por $72 \%$ das mortes ocorridas no País (SCHMIDT ET AL., 2011). Segundo a Organização Mundial de Saúde, os anos de vida perdidos ajustados por incapacidade - DALY (Disability-Adjusted Life Years - anos de vida perdidos por morte prematura e incapacidade) permitem estimar os efeitos das doenças crônicas de maneira mais adequada que apenas a mortalidade total. Um $D A L Y$ significa um ano de vida perdido (MURRAY; LOPEZ, 1996). Estudo realizado no Brasil sobre a carga de doença, com dados de 1998, já apontavam as doenças crônicas como responsáveis por $66,3 \%$ dos $D A L Y s$ (SCHRAMM; OLIVEIRA; LEITE, 2004).

A Sociedade Brasileira de Diabetes, em 2014, apontou que essa enfermidade atingiu mais de 387 milhões de pessoas em todo o mundo, correspondendo a $8,3 \%$ da população mundial. Apontou ainda que quase metade das pessoas com diabetes não foi sequer diagnosticada nas diversas nações do mundo contemporâneo. No Brasil, o diabetes esteve relacionado com a morte de mais de 48 mil pessoas entre 20 e 60 anos de idade, com uma prevalência estimada de $8,7 \%$.
Em dezembro de 2010, o Ministério da Saúde estabeleceu diretrizes para organização da Rede de Atenção à Saúde no âmbito do Sistema Único de Saúde (SUS) (BRASIL, 2010), e em 2013, instituiu a Rede de Atenção à Saúde das Pessoas com Doenças Crônicas (BRASIL, 2013). Posteriormente, estabeleceu diretrizes para a organização das linhas de cuidado da Rede de Atenção à Saúde das Pessoas com Doenças Crônicas (BRASIL, 2014A).

Os objetivos das RAS são melhorar a qualidade da atenção prestada e garantir a longitudinalidade do cuidado, por meio de medidas e ações articuladas de prevenção, promoção e tratamento. Adicionalmente, visa otimizar a utilização dos recursos e promover a equidade em saúde. Nesse sentido, busca promover a integralidade do cuidado com a oferta de um ciclo completo de atendimento ao usuário nos diferentes pontos de atenção da Rede. As RAS são compostas por: uma população específica, uma estrutura operacional e um modelo de atenção à saúde.

A população é o primeiro elemento constituinte das RAS, imprescindível para sua organização e operacionalização. O conhecimento em profundidade da população usuária de um sistema de saúde permite romper com a gestão baseada na oferta, característica dos sistemas fragmentados, e instituir a gestão baseada nas necessidades de saúde da população, elemento essencial das RAS.

A estrutura operacional é formada pela Atenção Primária à Saúde (APS), pela Atenção Especializada (AE), pelos Sistemas de Apoio Diagnóstico e Terapêutico (SADT), pelos Sistemas Logísticos (SL) e pelo Sistema de Governança (SG). A APS é o centro de comunicação da Rede e deve funcionar como ordenadora e coordenadora do cuidado. A $\mathrm{AE}$ reúne os diversos pontos de atenção com densidades tecnológicas distintas, como os ambulatórios, as emergências e os hospitais, devendo apoiar e complementar os serviços da atenção básica de forma resolutiva e em tempo oportuno. Os SADT ofertam o apoio no que diz respeito à diagnose e terapêutica: são 
os exames, as atividades corporais, as práticas integrativas e as atividades desempenhadas pelo Serviço de Atenção Domiciliar (SAD) e pelo Núcleo de Apoio à Saúde da Família (Nasf). Os SL são responsáveis pelo estabelecimento de ligações entre os pontos da rede, pela racionalidade de fluxos e contrafluxos de usuários, produtos e informações. O SG é responsável pelo acompanhamento da implantação da rede e articulação com os atores envolvidos (BRASIL, 2014A).

Por fim, o terceiro elemento constitutivo das RAS e fundamental para sua estruturação é o modelo de atenção à saúde. Ele define a forma como a atenção deve ser realizada na rede e como os diversos pontos devem se comunicar e se articular. O Modelo de Atenção às Condições Crônicas (MACC) foi criado no Brasil com o objetivo de nortear a atenção ao portador de doenças crônicas. Esse modelo prevê cinco níveis de intervenções em saúde: intervenções de promoção da saúde, de prevenção das condições de saúde, gestão da condição de saúde simples, gestão da condição de saúde complexa e gestão de caso para as condições de saúde muito complexas (BRASIL, 2014A; MENDES, 2012).

No Brasil, ainda existem poucos estudos acerca da temática de RAS na realidade do SUS. Entretanto, eles já apontam para uma inefetividade da APS em ser resolutiva, principal porta de entrada, ordenadora da Rede e coordenadora do cuidado (ALMEIDA ET AL., 2010; AZEVEDO, COSTA, 2010; GIOVANELLA; ESCOREL; MENDONÇA, 2003). Apontam também para ineficiência da $\mathrm{AE}$ em apoiar e complementar os serviços da atenção básica de forma resolutiva e em tempo oportuno (OLIVEIRA ET AL., 2010; SILVA, 2012; SPEDO; PINTO; TANAKA, 2010). Entretanto, pouco ainda se tem refletido sobre a implantação dessas redes. Hartz e Contandriopoulos (2004) afirmam que o questionamento acerca da implantação deve ser anterior ao da eficiência ou efetividade.

Dessa forma, o objetivo deste artigo é avaliar a implantação da RAS aos portadores de Diabetes Mellitus no SUS em Recife
(PE), incluindo a análise da estrutura e do processo de todos os seus componentes, bem como a análise de fatores contextuais que influenciam o seu grau de implantação. Isso permite identificar entraves e proposições para sua implantação. A identificação de tais aspectos, realizada em uma pesquisa desenvolvida em um distrito sanitário de Recife, é relevante pela possibilidade de contribuir na reorganização dessa rede, com vistas ao seu aperfeiçoamento e melhora da qualidade do serviço ofertado.

\section{Métodos}

Foi realizada uma pesquisa avaliativa do tipo análise de implantação (CHAMPANGNE, 2011), utilizando o modelo Político Contingente, proposto por Denis e Champangne (1997), para análise do contexto. Foi realizado o estudo de caso da RAS aos portadores de Diabetes Mellitus (DM) do município de Recife, tomando por referência o Distrito Sanitário III. O distrito escolhido dispõe de tecnologias de distintas complexidades que visam assegurar a oferta do atendimento integral.

Para avaliação do grau de implantação, foram construídos o Modelo Lógico da intervenção (figura 1), a Matriz de Análise e Julgamento e avaliadas as dimensões de estrutura e processo dessa RAS. Na dimensão estrutura, foram avaliados o espaço físico, os recursos humanos e os equipamentos e materiais necessários para oferta do atendimento integral ao portador de DM na RAS, por meio de 55 indicadores. Na dimensão processo, foram avaliadas as atividades pertinentes a cada componente, que em conjunto possibilitaria a oferta da atenção integral ao portador de DM na RAS. Essas atividades foram avaliadas por meio de 60 indicadores (SANTOS, 2015). O modelo lógico e a matriz de análise e julgamento foram submetidos à apreciação de especialistas, conforme recomendação de McLaughlin e Jordan (1999). 
Figura 1. Modelo Lógico da Rede de Atenção à Saúde ao portador de Diabetes Mellitus.

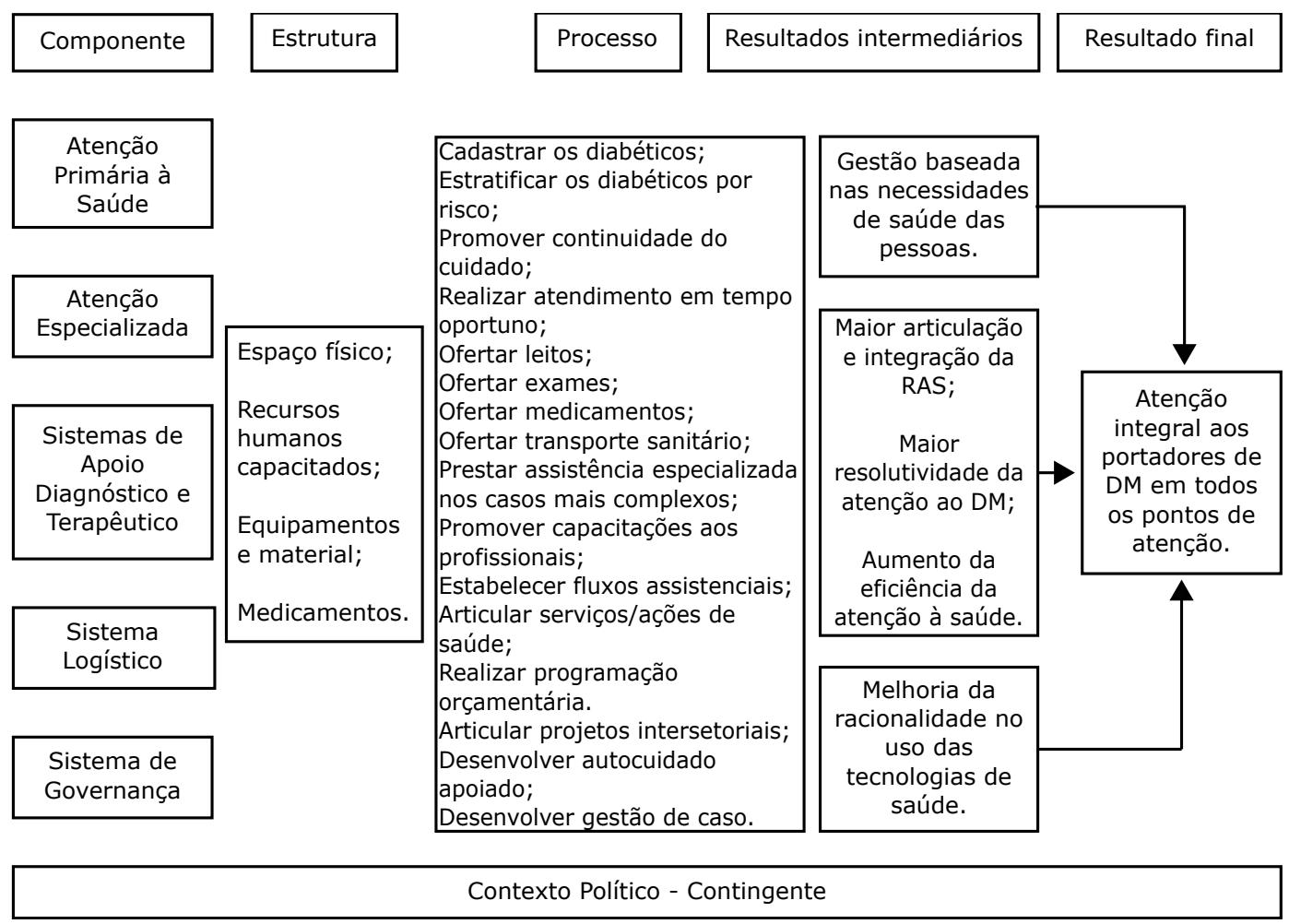

Fonte: Elaboração própria.

Para avaliação do contexto, foi construída sua matriz de análise. Nessa matriz, foram analisadas três variáveis relacionadas ao contexto estrutural (atributos operacionais, dos gestores e do ambiente) e três relacionadas ao contexto político (suporte dado à intervenção, controle na organização para operacionalizar a intervenção e coerência entre os objetivos da implantação da RAS e as ações). Ao total, foram criadas dez categorias de análise.
Após a determinação do contexto, foiverificada a sua influência sobre o grau de implantação.

Foram realizadas entrevistas estruturadas e semiestruturadas, observação direta e análise documental dos Planos Municipais de Saúde - 2010-2013 e 2014-2017 - e da Programação Anual de Saúde de 2013 (quadro 1). Na consulta a documentos, buscou-se identificar aspectos relacionados à Atenção à Saúde dos portadores de doenças crônicas e DM e sua respectiva RAS. 
Quadro 1. Síntese dos métodos de coleta e das fontes de informações utilizadas na etapa de análise da implantação segundo os componentes

\begin{tabular}{|c|c|c|c|}
\hline Componente & $\begin{array}{l}\text { Método de } \\
\text { Coleta }\end{array}$ & Fonte de Informação & $\begin{array}{l}\text { Etapa de análise da } \\
\text { implantação }\end{array}$ \\
\hline $\begin{array}{l}\text { Atenção Primária à } \\
\text { Saúde }\end{array}$ & $\begin{array}{l}\text { Entrevista } \\
\text { estruturada }\end{array}$ & Informante-chave (médico/enfermeiro) & Determinação do Gl \\
\hline Atenção Especializada & $\begin{array}{l}\text { Entrevista } \\
\text { estruturada }\end{array}$ & Informante-chave (médico/enfermeiro) & Determinação do Gl \\
\hline $\begin{array}{l}\text { Sistema de Apoio } \\
\text { Diagnóstico e } \\
\text { Terapêutico }\end{array}$ & $\begin{array}{l}\text { Entrevista } \\
\text { estruturada }\end{array}$ & $\begin{array}{l}\text { Informante-chave (trabalhadores da rede - } \\
\text { APS, AE e dos SADT) }\end{array}$ & Determinação do Gl \\
\hline \multirow{4}{*}{$\begin{array}{l}\text { Sistema de Apoio } \\
\text { Logístico }\end{array}$} & $\begin{array}{l}\text { Entrevista } \\
\text { estruturada }\end{array}$ & $\begin{array}{l}\text { Informante-chave (trabalhadores da rede - } \\
\text { APS, AE e dos SADT) }\end{array}$ & Determinação do Gl \\
\hline & $\begin{array}{l}\text { Entrevista } \\
\text { semiestruturada }\end{array}$ & $\begin{array}{l}\text { Informante-chave } \\
\text { (Secretária Executiva de Regulação e Saúde) }\end{array}$ & $\begin{array}{l}\text { Estudo do contexto de } \\
\text { implantação }\end{array}$ \\
\hline & $\begin{array}{l}\text { Entrevista } \\
\text { estruturada }\end{array}$ & $\begin{array}{l}\text { Informantes-chave } \\
\text { (Coordenadora da Política de Saúde dos }\end{array}$ & Determinação do Gl \\
\hline & $\begin{array}{l}\text { Entrevista } \\
\text { semiestruturada }\end{array}$ & $\begin{array}{l}\text { Portadores de DCNT, Secretária Executiva } \\
\text { de Regulação e Saúde, e Coordenadora da } \\
\text { Vigilância Epidemiológica Distrital) }\end{array}$ & $\begin{array}{l}\text { Estudo do contexto de } \\
\text { implantação }\end{array}$ \\
\hline \multirow[t]{2}{*}{ Sistema de Governança } & $\begin{array}{l}\text { Observação } \\
\text { direta }\end{array}$ & $\begin{array}{l}\text { Existência de setor específico para RAS aos } \\
\text { portadores de doenças crônicas e condições } \\
\text { de trabalho no Nível Central. }\end{array}$ & $\begin{array}{l}\text { Estudo do contexto de } \\
\text { implantação }\end{array}$ \\
\hline & $\begin{array}{l}\text { Consulta a } \\
\text { documentos }\end{array}$ & $\begin{array}{l}\text { Planos Municipais de Saúde (2010-2013 e } \\
\text { 2014-2017) e Programação Anual de Saúde } \\
\text { (2014) }\end{array}$ & $\begin{array}{l}\text { Estudo do contexto de } \\
\text { implantação }\end{array}$ \\
\hline
\end{tabular}

Fonte: Elaboração própria.

Nota: GI: Grau de implantação; APS: Atenção Primária à Saúde; AE: Atenção Especializada; SADT: Sistemas de Apoio Diagnóstico e

Terapêutico; DCNT: Doenças Crônicas Não Transmissíveis.

Nos diferentes níveis de complexidade da RAS, no período de outubro de 2014 a janeiro de 2015 , foram entrevistados 22 profissionais, reconhecidos como informantes-chave nos serviços analisados. Foi considerada informante-chave a pessoa que possuía conhecimento especial sobre o que se pretendia averiguar para compartilhar com o entrevistador (TOBAR; YALOUR, 2001).

Esses profissionais estavam distribuídos da seguinte forma: seis profissionais médicos ou enfermeiros nas Unidades de Saúde da Família (USF), representando a totalidade do componente da APS; cinco profissionais médicos ou enfermeiros na AE (ambulatórios, emergências e hospital); oito profissionais enfermeiros, educadores físicos ou fisioterapeutas no Sistema de Apoio Diagnóstico e Terapêutico (Nasf, SAD e Programa Academia de Saúde); a Secretária Executiva de Regulação em Saúde da Secretaria Municipal de Saúde (SMS), pertencente ao Sistema Logístico; a coordenadora de Vigilância Epidemiológica do distrito sanitário e a coordenadora da Política de Saúde dos Portadores de DCNT da SMS, pertencente ao Sistema de Governança da Rede. Todos os serviços da AE são referência para o tratamento do DM no município.

Para análise do grau de implantação das dimensões estrutura e processo, foi realizado o somatório das pontuações obtidas nos indicadores de cada dimensão 
separadamente, dividido pela pontuação esperada para a dimensão, e depois multiplicado por 100. O grau de implantação total (estrutura + processo) foi estabelecido de forma ponderada, por considerar que a dimensão processo era mais importante que a dimensão estrutura na implantação da RAS aos portadores de DM. Nesse sentido, foi atribuído peso quatro para a dimensão estrutura e peso seis para a dimensão processo. Assim, o grau de implantação (GI) total de cada unidade de análise foi obtido da seguinte forma ${ }^{\mathbf{1}}$ :

$$
\text { GI Total }=\left(\frac{\left(4 \sum E^{1}+6 \sum P^{1}\right) / 10}{\left(4 \sum E^{1}+6 \sum P^{2}\right) / 10}\right) * 100
$$

A análise considerou os seguintes pontos de corte: $<25,0 \%$ - não implantado; de $25,1 \%$ a $50,0 \%$ - implantação incipiente; de $50,1 \%$ a $75,0 \%$ - parcialmente implantado; e $>75,1 \%$ - implantado.

O material resultante das entrevistas semiestruturadas e da análise documental foi submetido à análise de conteúdo temática à luz do modelo Político-Contingente para avaliação do contexto. Esse foi considerado favorável quando o número de categorias temáticas favoráveis era maior que o de desfavoráveis.

Para avaliação da influência do contexto sobre o grau de implantação, foi feito o confronto da classificação do GI obtido com a classificação final do contexto político de gestão.

A pesquisa obedeceu às normas que regem as pesquisas com seres humanos, obtendo aprovação da Comissão Nacional de Ética e Pesquisa (Conep) sob parecer 768.025 e do Comitê de Ética e Pesquisa da Fundação Oswaldo Cruz Pernambuco sob parecer 421.616.

\section{Resultados}

\section{Grau de implantação}

A implantação da RAS aos portadores de DM foi classificada como de implantação parcial (GI $=65,5 \%$ ), considerando as dimensões estrutura e processo. A dimensão estrutura (GI de 58,1\%) obteve pior desempenho que a dimensão processo (73,1\%). À exceção do Sistema Logístico, para o qual se observou implantação incipiente (GI=46,9\%), todos os demais componentes da RAS estavam parcialmente implantados (tabela 1).

Tabela 1. Grau de implantação (\%) geral e por dimensões de estrutura e processo da Rede de Atenção à Saúde ao portador de DM no Distrito Sanitário de Recife segundo os componentes, 2014-2015.

\begin{tabular}{|c|c|c|c|c|c|c|}
\hline Dimensões & APS & $\mathrm{AE}$ & SADT & SL & SG & DS \\
\hline Estrutura & 48,8 & 62,0 & 68,2 & 45,5 & 90,0 & 58,1 \\
\hline Processo & 72,6 & 77,2 & 72,0 & 60,0 & 54,5 & 73,1 \\
\hline Grau de Implantação Ponderado & 63,8 & 69,4 & 69,2 & 46,9 & 67,9 & 65,5 \\
\hline
\end{tabular}

Fonte: Elaboração própria.

Nota: APS: Atenção Primária à Saúde; AE: Atenção Especializada; SADT: Sistemas de Apoio Diagnóstico e Terapêutico; SL: Sistema Logístico; SG: Sistema de Governança; DS: Distrito Sanitário.

\section{Avaliação da dimensão estrutura}

Na avaliação realizada para o conjunto dos componentes da APS, a dimensão estrutura caracterizou-se como de implantação incipiente (tabela 1). O espaço físico foi considerado adequado, com GI $=83,3 \%$, porém os recursos humanos obtiveram implantação parcial (GI=58,3\%). Foi verificado que o apoio matricial às Equipes de Saúde da Família (EqSF) não era realizado na frequência considerada adequada
1 Em que $\sum \mathrm{E}^{1}=$ Somatório das pontuações obtidas nos indicadores que compunham a dimensão estrutura; $\sum \mathrm{P}^{1}=$ Somatório das pontuações obtidas nos indicadores que compunham a dimensão processo; $\sum \mathrm{E}^{2}=$ Somatório das pontuações esperadas nos indicadores que compunham a dimensão estrutura; $e$ $\sum \mathrm{P}^{2}=$ Somatório das pontuações esperadas nos indicadores que compunham a dimensão processo. 
( $\mathrm{GI}=25 \%$ ). Quanto aos equipamentos e materiais, estes foram classificados como grau de implantação incipiente, em relação ao material necessário ao cadastramento dos diabéticos (GI=33\%), e não implantados no que se refere aos protocolos para estratificação dos diabéticos por risco (GI=16,7\%). As medicações, avaliadas quanto à disponibilidade e regularidade na distribuição, foram classificadas desde não implantadas (para controle de neuropatias e fitoterápicos, homeopáticos ou plantas medicinais), com implantação incipiente (para melhora do perfil lipídico), até parcialmente implantadas (para controle de DM, controle pressórico e medicação cardioprotetora).

$\mathrm{Na} \mathrm{AE}$, a dimensão estrutura obteve $\mathrm{GI}=62 \%$, sendo, portanto, classificada como parcialmente implantada (tabela 1). O espaço físico foi considerado parcialmente implantado (GI $=65 \%$ ), os recursos humanos foram caracterizados como existindo em quantidade suficiente (GI=80\%), porém ainda sem a qualificação necessária ao desenvolvimento de suas atividades (GI $=40 \%)$. Quanto aos equipamentos e materiais, destaca-se a inexistência de protocolos (para atendimento do DM, para estratificação por risco, para solicitação de exames) nos serviços de urgência e emergência $(\mathrm{GI}=0 \%)$. As medicações foram caracterizadas desde implantação incipiente (fitoterápicos, homeopáticos ou plantas medicinais), parcialmente implantadas (para controle do DM e de neuropatias), até implantadas (para controle pressórico, cardioprotetora e para melhora do perfil lipídico).

Nos SADT, a dimensão estrutura caracterizou-se como parcialmente implantada (tabela 1), com destaque para a existência de referência para o Nasf (GI=100\%) e a não existência de referência sistemática para clínica especializada em tempo oportuno (GI=48,5\%). A disponibilização de exames também foi considerada parcialmente implantada.
Já o Sistema Logístico obteve o pior desempenho da dimensão estrutura (tabela 1) por dispor de maneira insatisfatória da maior parte dos recursos necessários ao desenvolvimento de suas atividades. O transporte sanitário caracterizou-se com implantação incipiente (GI=36,4\%) devido à inexistência de um transporte próprio da Unidade, embora existisse apoio do Sistema de Atendimento Móvel de Urgência (Samu). O prontuário eletrônico único ou outro mecanismo que garantisse a continuidade informacional foi caracterizado como não implantado (GI=0\%). Apenas a Central de marcação de consultas, exames e regulação de leitos foi considerada implantada (GI $=100 \%)$.

O componente da RAS como um todo que apresentou o melhor desempenho em relação à estrutura foi o Sistema de Governança (tabela 1). Esse componente foi caracterizado com adequado espaço físico e equipamentos e materiais necessários ao desenvolvimento de suas atividades: local de trabalho, acesso a documentos e disponibilidade de recursos financeiros (GI $=100 \%)$. Os recursos humanos, entretanto, obtiveram GI incipiente (GI $=50 \%$ ), devido à quantidade de profissionais classificada como insuficiente para a realização das atividades.

\section{Avaliação da dimensão processo}

Na APS, destacaram-se as atividades de cadastro e de estratificação por risco, que se caracterizaram como não implantadas. O encaminhamento para outros níveis de atenção foi considerado implantado em todas as EqSF, mas a contrarreferência, analisada pelo indicador 'comunicação entre pontos de atenção' caracterizou-se como não implantada, tendo sido relatada acontecer nunca ou quase nunca por $66,7 \%$ das EqSF analisadas, e raramente ou às vezes pelo restante das equipes.

$\mathrm{Na} \mathrm{AE}$, a consulta caracterizou-se como não implantada $(\mathrm{GI}=0 \%)$ porque não era 
realizada na frequência recomendada pelo Ministério da Saúde nem era realizada em sua completude. Também foram considerados não implantados: a comunicação entre os pontos de atenção nos ambulatórios (GI=25\%) e a discussão de caso clínico nos hospitais (GI $=0 \%)$.

Nos SADT, as atividades de matriciamento e gestão compartilhada obtiveram GI incipiente. Todas as outras atividades desse componente caracterizaram-se como parcialmente implantadas (visita domiciliar, atendimento domiciliar, atividades corporais) ou implantadas (suporte técnico e emocional, educação em saúde, projeto terapêutico singular e projeto saúde no território).

O Sistema Logístico caracterizou-se como incipiente quanto à regulação de leitos (GI=50\%) e parcialmente implantado quanto ao agendamento de consultas e exames (GI=66,7\%). Já no Sistema de Governança, foi possível observar a não implantação para programação orçamentária e análise e divulgação de informações e implantação incipiente para articulação de serviços de saúde. As demais atividades foram consideradas implantadas (construção de diagnósticos, estabelecimento de prioridades, monitoramento e avaliação).

\section{Análise do contexto e sua influência sobre o grau de implantação}

O contexto apresentou-se favorável à implantação da RAS ao portador de DM no Distrito Sanitário de Recife. Obteve 50\% das categorias no contexto político e $83,3 \%$ no contexto estrutural, perfazendo um total de $66,7 \%$ de categorias favoráveis.

A coerência do plano municipal de saúde (2014-2017) com a implementação da RAS ao portador de DM mantém relação positiva com alguns achados na avaliação do GI. São exemplos: nos SADT, a existência de referência para o SAD, para o Nasf; no sistema de Governança, a construção de diagnósticos, o monitoramento e a avaliação das ações relacionadas à atenção à saúde dos portadores de DM, entre outras. Também contribui positivamente para a implantação da RAS a existência de um setor específico para a Coordenação da Política de Saúde dos portadores de DCNT no município.

Alguns aspectos do contexto, entretanto, influenciam negativamente a implantação da RAS aos portadores de DM no distrito. $\mathrm{O}$ fato de a Coordenação da Política de Saúde das pessoas com doenças crônicas ter sido criada há pouco tempo (menos de dois meses quando da realização da pesquisa) demonstra que esta é uma preocupação recente no município, e esse aspecto tem intrínseca relação com a falta de programação orçamentária, de análise e divulgação de informações acerca da situação de saúde dos portadores de DM. Esse fato também pode ter corroborado a frágil comunicação existente entre os diferentes pontos de atenção da Rede.

A inexistência de um responsável pela Vigilância Epidemiológica (VE) das DCNT no distrito ocasionou dificuldades nas análises e divulgação de informações pela Governança. A inexistência do prontuário eletrônico único ou outro mecanismo que garantisse a comunicação entre os pontos de atenção à saúde foi relacionada à pouca prioridade dada à temática pela gestão.

\section{Discussão}

Os resultados que serão aqui discutidos são parte integrante de um estudo mais amplo que objetiva avaliar como estão inseridos os componentes de alimentação, nutrição, atividade física e assistência farmacêutica na rede de atenção ao portador de doenças crônicas, a partir da Estratégia Saúde da Família em quatro polos de macrorregiões de Pernambuco. Para tanto, tornou-se necessária a identificação da existência da própria Rede. A discussão que se inicia 
debate acerca dos resultados encontrados para implantação dessa RAS.

No processo de estruturação e organização, ou seja, na institucionalização de uma RAS, alguns elementos são essenciais. População, modelo de atenção e serviços de saúde precisam estar interligados a fim de que se possa romper com a gestão baseada na oferta, característica dos sistemas fragmentados, e instituir a gestão baseada nas necessidades de saúde da população, garantindo a longitudinalidade do cuidado e a integralidade da atenção. Nesse sentido, a população de responsabilidade da RAS deve ser totalmente conhecida e estratificada em subpopulações por fatores de risco (MENDES, 2012).

No que se refere à RAS ao portador de DM, as ações de cadastro e de estratificação por risco foram caracterizadas como não implantadas. Corroboram a não realização dessas ações a inexistência de protocolos para estratificação dos pacientes por risco, verificada em $83,3 \%$ das USF estudadas, e a implantação incipiente de material para cadastro dos diabéticos.

Apesar de ser uma ferramenta útil e necessária, o protocolo de estratificação de risco isoladamente não é capaz de definir o risco ou a vulnerabilidade do indivíduo. O Ministério da Saúde destaca que o diálogo, a interação, a escuta, o respeito e o acolhimento do usuário e sua queixa são necessários para avaliação do potencial de agravamento (BRASIL, 2012). Essas atitudes não só balizam a estratificação dos indivíduos como também contribuem para aumentar a resolubilidade da APS.

Nessa perspectiva, atividades de matriciamento, gestão compartilhada, projetos intersetoriais, entre outros, são estimuladas para serem adotadas nos serviços de saúde pelo Ministério da Saúde (BRASIL, 2014B). No presente estudo, todas essas atividades obtiveram GI incipiente, o que já corrobora uma baixa resolubilidade desse nível de atenção.

No que se refere à disponibilidade e regularidade de distribuição das medicações, essencial para o controle do DM e suas comorbidades, observa-se que a quantidade de medicamentos recebidos pelos serviços de saúde não corresponde à necessidade de todos os usuários. Situações semelhantes foram verificadas por Souza et al. (2008) e Serapioni e Silva (2011), demonstrando a necessidade de uma observação mais criteriosa acerca da Assistência Farmacêutica que vem sendo realizada no Brasil.

Ainda como prerrogativa de funcionamento da Rede de Atenção à Saúde ao portador de DM, a APS deve funcionar como ordenadora e coordenadora do cuidado. Nesse sentido, para que as necessidades de saúde sejam atendidas nos espaços tecnológicos mais adequados e para que a APS garanta a continuidade da atenção, ela deve realizar encaminhamentos para consultas com especialistas, realização de exames ou ainda para práticas corporais e/ ou integrativas, sempre que detectada necessidade (TANAKA, 2011).

Dessa forma, o retorno desses encaminhamentos (a contrarreferência) é essencial para o exercício da coordenação da atenção pela APS. É essa comunicação entre os pontos de atenção da rede que darão racionalidade ao atendimento, evitando que consultas e exames sejam realizados em duplicidade ou que sejam solicitados desnecessariamente.

O encaminhamento para outros níveis de atenção foi considerado implantado em todas as EqSF estudadas. Um importante fator que contribuiu para isso foi a existência e utilização do fluxograma de atendimento ao DM verificadas no município. Giovanella, Escorel e Mendonça (2003) consideram o estabelecimento de fluxos uma importante estratégia para a integração do sistema e melhoria da referência.

Já a contrarreferência foi caracterizada como não implantada tanto pela APS quanto pela AE. Esses resultados estão em consonância com o estudo de Fausto et al. (2014), que verificaram que $52 \%$ dos especialistas para quem os pacientes são encaminhados nunca entram em contato com a 
EqSF para trocar informações. No mesmo sentido, Giovanella, Escorel e Mendonça (2003) observaram que um terço ou mais dos profissionais de nível superior das EqSF nunca recebem qualquer tipo de contrarreferência de pacientes atendidos na $\mathrm{AE}$.

A implantação do cartão do usuário do SUS é uma possível estratégia para garantir a contrarreferência e melhorar a articulação entre os serviços de saúde (GIOVANELLA; ESCOREL; MENDONÇA, 2003). Medina (2006), entretanto, acredita que os problemas concernentes à comunicação estão mais relacionados à atuação do profissional de saúde - seu engajamento, concepções e disposições - que à existência de ferramentas gerenciais. A autora observou que a disponibilidade de instrumentos de comunicação (guias de referência e contrarreferência e telefone) não influenciou nos escores relacionados a essa questão.

Assim, constata-se a necessidade de investir na formação dos profissionais de saúde que trabalham ou trabalharão no SUS, para que eles tenham uma prática adequada aos preceitos desse sistema. É fundamental que eles reconheçam a importância da realização da contrarreferência e de seu papel na corresponsabilização da saúde de seu paciente.

Outro mecanismo que visa estabelecer a continuidade informacional acerca da situação de saúde dos usuários é o prontuário eletrônico único, que se caracterizou como não implantado neste estudo, não tendo sido observado em qualquer serviço de saúde analisado. Um estudo realizado em 2012 com 16.566 EqSF demonstra que essa é a realidade vivenciada pela maioria das equipes de saúde no País, visto que apenas $11 \%$ das equipes relataram utilizar o prontuário eletrônico único no seu cotidiano (FAUSTO ET AL., 2014).

A referência para clínica especializada caracterizou-se com implantação incipiente por não ofertar cotas para todas as especialidades em quantidade suficiente e as consultas não ocorrerem em tempo oportuno. Situações semelhantes a essa foram observadas em outras cidades, como Florianópolis (SISSON ET AL., 2011) e uma cidade do estado de São Paulo (MARIN; MARCHIOLl; MORACVICK, 2013). Para os autores, o fato dos serviços de maior densidade tecnológica não terem acompanhado a ampliação da cobertura da Estratégia de Saúde da Família ocasionou na descontinuidade da atenção e em prejuízos aos atendimentos dos usuários.

Muitos autores concordam que a resolubilidade da atenção básica depende, em grande medida, do acesso aos serviços especializados (ESCOREL ET AL., 2007) e que essa dificuldade de acesso tem se revelado um dos principais entraves na garantia da integralidade da atenção (SPEDO; PINTO; TANAKA, 2010), condição indispensável para efetiva implantação das RAS.

Esse fato sugere que a simples ampliação da cobertura dos serviços especializados pudesse garantir a integralidade da atenção. Entretanto, em 2008, no município de Recife, as cinco especialidades com maior demanda reprimida (oftalmologia, ortopedia, otorrinolaringologia, cardiologia e urologia) eram as mesmas com maiores percentuais de absenteísmo nas consultas. Dessa forma, ou existe uma real insuficiência na oferta, ou existe um mau gerenciamento para esses procedimentos, em que não se realiza o diagnóstico consistente da capacidade instalada e o percentual efetivo de utilização desses serviços (ALbUQUeRQUE ET AL., 2013). Para Alves (2011), a demora na obtenção do atendimento pode levar o usuário agendado a faltar, uma vez que ele não poderia esperar tanto tempo pelo procedimento e procuraria outros serviços.

Portanto, fica evidenciada a necessidade de aperfeiçoamento do processo de regulação desses procedimentos. Quanto a essa questão, o contexto apresentou-se favorável. O município opera com o sistema de regulação on-line disponibilizado pelo Departamento de Informática do SUS (Datasus), o Sisreg, que permite ao gestor conhecer o tamanho real das filas de espera, monitorá-las, definir prioridades 
clínicas, planejar a oferta da AE e monitorar o índice de abstenção a consultas e exames (ALMEIDA ET AL., 2010).

Além disso, a regulação municipal capacita os profissionais de nível superior e técnico acerca dos mecanismos de marcação de consultas e exames, priorizando sempre os serviços mais próximos da localidade do usuário no momento da marcação. Também está em processo de implantação o Short Message Service $(S M S)$, que informa ao usuário o local, a data e o horário da consulta via telefonia móvel (aparelho celular), sempre que ele é agendado. O serviço de Call Center é disponibilizado para os usuários retirarem dúvidas. Todos esses mecanismos visam reduzir o absenteísmo, ordenar fluxos e reduzir o tempo de espera.

O município de Recife é habilitado em gestão plena. Segundo a Norma Operacional de Assistência à Saúde (Noas) 2002 e o Pacto pela Saúde 2006, nesta condição, um único gestor responde por toda a rede assistencial na sua área de abrangência, conduz a negociação com os prestadores e assume o comando da política de saúde em seu território.

No período da pesquisa, o leito hospitalar e alguns exames mais complexos (como cintilografia, ressonância) permaneciam sendo regulados pelo Estado, e a regulação de leitos foi caracterizada com GI incipiente por não ocorrer em tempo oportuno. Apesar disso, o contexto foi julgado favorável à implantação porque além de o município ter investido nos mecanismos de SMS e Call Center, encontrava-se em fase final a construção de um hospital municipal que disponibilizaria leitos e exames de alta complexidade, totalmente regulado pelo município. Além disso, existia a perspectiva de o município assumir a totalidade de sua regulação, assumindo uma Central Regional, em que passaria a regular os serviços de saúde de alta complexidade para mais 71 municípios.

O transporte sanitário, importante mecanismo do Sistema Logístico por sua responsabilidade de garantir o fluxo de pessoas na RAS, foi caracterizado com GI incipiente, tendo sido relatado existir apenas nas unidades sob gestão estadual. Algumas unidades sob gestão municipal afirmaram disponibilizar vale-transporte em algumas situações ou, ainda, contatar o Samu em caso de urgências e emergências.

Um estudo realizado na cidade de Belo Horizonte acerca do funcionamento do transporte sanitário de pessoas demonstrou que esse serviço integrado às várias unidades de saúde de diferentes níveis de complexidade foi decisivo para atender às necessidades dos usuários. Antes, estes eram encaminhados e necessitavam se deslocar com recursos próprios, de que nem sempre dispunham. Com o serviço, possibilitou-se o acesso e, consequentemente, a maior satisfação dos usuários (ALVES; SILVA; NEIVA, 2010).

A governança, que possui papel fundamental na implantação da RAS ao portador de DM, caracterizou-se por não realizar a programação orçamentária nem a análise e divulgação de informações de maneira satisfatória. Segundo Vieira (2009), o planejamento é vital para que se alcance os resultados esperados. Sem ele, as atividades são realizadas por inércia, de forma desarticulada e desordenada, e os recursos são utilizados de forma menos eficiente.

Chama a atenção o tempo de implantação da Coordenação da Política de Saúde das pessoas com DCNT no município (inferior a dois meses no período do estudo). Esse fato, ao mesmo tempo em que demonstra que a saúde das pessoas portadoras de doenças crônicas é uma prioridade recente da gestão, também justifica a não realização de algumas atividades por essa Coordenação, haja vista o seu curto período de estruturação.

A existência de uma equipe qualificada, porém com profissionais em quantidade insuficiente nessa Coordenação, reforça os achados. A equipe, que era composta pela Coordenadora da Política e por mais quatro apoiadores institucionais com formação 
em saúde pública, foi considerada pequena para atender às demandas do Sistema de Governança. Cada apoiador ficava responsável sozinho por dois distritos sanitários e, além da política de DCNT, também era responsável por outras políticas. Dessa forma, apesar de possuírem qualificação, o acúmulo de responsabilidades não permitia que desenvolvessem suas atividades de maneira satisfatória.

No quadro 2, são apresentadas as principais dificuldades relacionadas à implementação da RAS ao portador de DM e algumas proposições baseadas na equidade, na integralidade e na qualificação da gestão, visando à implementação da RAS.

Quadro 2. Problemas e proposições relacionadas à implementação da Rede de Atenção à Saúde ao portador de Diabetes Mellitus. Recife, 2014-2015

\begin{tabular}{|c|c|c|}
\hline Componente & Limitações à implementação da RAS & Proposições para implementação da RAS \\
\hline APS & $\begin{array}{l}\text { - Material para cadastro dos diabéticos em quantidade } \\
\text { insuficiente; } \\
\text { - Inexistência de protocolo para estratificação dos } \\
\text { diabéticos por risco; } \\
\text { - Não realização do cadastro dos diabéticos; } \\
\text { - Não estratificação dos diabéticos por riscos; }\end{array}$ & $\begin{array}{l}\text { Objetivo: Garantir a equidade, tendo a população como essência } \\
\text { da RAS } \\
\text { - Aproximar VE com o cenário de ocorrência das DCNT, com } \\
\text { vistas a fortalecer a estratificação por riscos e, assim, garantir a } \\
\text { gestão baseada nas necessidades de saúde da população; } \\
\text { - Ampliar a oferta de material, inclusive para cadastro e protocolo } \\
\text { de estratificação por risco; }\end{array}$ \\
\hline $\mathrm{AE}$ & $\begin{array}{l}\text { - Não realização da contrarreferência, sugerindo para } \\
\text { uma prática de desresponsabilização dos profissionais } \\
\text { com a saúde do paciente; } \\
\text { - Deficiências na organização do sistema de } \\
\text { contrarreferência à APS; } \\
\text { - Inexistência de protocolos e manuais; }\end{array}$ & $\begin{array}{l}\text { Objetivo: Garantir a integralidade da atenção, com foco na } \\
\text { responsabilização dos profissionais de saúde } \\
\text { - Ofertar educação permanente para os profissionais de saúde que } \\
\text { atuam no SUS para que eles tenham uma prática harmônica aos } \\
\text { preceitos do sistema; } \\
\text { - Organizar o sistema de contrarreferência à APS; } \\
\text { - Ofertar protocolos e manuais; }\end{array}$ \\
\hline SADT & $\begin{array}{l}\text { - Incipiente realização das atividades matriciais e gestão } \\
\text { compartilhada entre Nasf/SAD e APS; }\end{array}$ & $\begin{array}{l}\text { Objetivo: Garantir a integralidade, com foco na resolubilidade da } \\
\text { atenção } \\
\text { - Definir responsabilidades de cada serviço; } \\
\text { - Aumentar a interlocução entre os serviços; } \\
\text { - Incrementar o apoio matricial à APS para aumentar a } \\
\text { resolubilidade desse componente; }\end{array}$ \\
\hline SL & $\begin{array}{l}\text { - Fragilidade na comunicação entre os pontos de atenção } \\
\text { da Rede; } \\
\text { - Ausência de transporte sanitário no nível municipal; } \\
\text { - Regulação sob duplo comando; }\end{array}$ & $\begin{array}{l}\text { Objetivo: Garantir a equidade e a integralidade da atenção através } \\
\text { da qualificação da gestão } \\
\text { - Implementar mecanismos que garantam a comunicação entre os } \\
\text { pontos de atenção; } \\
\text { - Padronizar as informações de preenchimento obrigatório } \\
\text { no prontuário eletrônico único e instituí-lo como meio de } \\
\text { avaliação dos serviços de saúde, com vistas ao fortalecimento da } \\
\text { continuidade informacional; } \\
\text { - Efetivar a regulação sob comando único; } \\
\text { - Ofertar o transporte sanitário no nível municipal e articular com } \\
\text { a AS o transporte daqueles que não dispõem de recursos; }\end{array}$ \\
\hline SG & $\begin{array}{l}\text { - Deficiência na organização do processo de gestão; } \\
\text { - Equipe em quantidade insuficiente; } \\
\text { - Não realização da programação orçamentária; } \\
\text { - Não realização da análise e divulgação de informações; }\end{array}$ & $\begin{array}{l}\text { Objetivo: Qualificar a estrutura e o processo de trabalho da gestão } \\
\text { - Apoiar e reforçar a Coordenação da Política de Saúde dos } \\
\text { portadores de DCNT no município, com vistas a melhorar a } \\
\text { atenção aos pacientes diabéticos. } \\
\text { - Aumentar a quantidade de profissionais da equipe; }\end{array}$ \\
\hline
\end{tabular}

Fonte: Elaboração própria.

Nota: APS: Atenção Primária à Saúde; AE: Atenção Especializada; SADT: Sistemas de Apoio Diagnóstico e Terapêutico; SL: Sistema Logístico; SG: Sistema de Governança; RAS: Rede de Atenção à Saúde; DM: Diabetes Mellitus; Nasf: Núcleo de Apoio à Saúde da Família; SAD: Serviço de Atenção Domiciliar; VE: Vigilância Epidemiológica; DCNT: Doença Crônica Não Transmissível; AS: Assistência Social. 
A todas essas proposições, acrescente-se a importância do mútuo funcionamento de todos os componentes da Rede, dada a interdependência que possuem. Só assim, a implementação da RAS ao portador de DM será plena.

\section{Conclusão}

Foi verificada a existência de dificuldades relacionadas à estrutura e ao processo de trabalho nos diferentes níveis de atenção à saúde (atenção primária, AE e nos SADT) e de gestão (Sistemas Logístico e de Governança).

\section{Referências}

ALBUQUERQUE, M. S. V. et al. Regulação assistencial no recife: possibilidades e limites na promoção do acesso. Saúde e sociedade, São Paulo, v. 22, n. 1, p. 223-236, mar. 2013.

ALMEIDA, P. F. et al. Desafios à coordenação dos cuidados em saúde: estratégias de integração entre níveis assistenciais em grandes centros urbanos. Cadernos de Saúde Pública, Rio de Janeiro, v. 26, n. 2, p. 286-298, fev. 2010.

ALVES, P. C. Desafios à integração do SUS: uma análise da rede de atenção à saúde da mulher em Recife. 2011. 150 f. Dissertação (Mestrado em Saúde Pública) - Centro de Pesquisas Aggeu Magalhães, Fundação Oswaldo Cruz, Recife, 2011

ALVES, M.; SILVA, A. F.; NEIVA, L. C. F. Atendimento de urgência: o transporte sanitário como observatório de saúde de Belo Horizonte. Revista Mineira de Enfermagem, Belo Horizonte, v. 14, n. 2, p. 181-187, abr./jun. 2010.

AZEVEDO, A. L. M.; COSTA, A. M. A estreita porta de entrada do Sistema Único de Saúde (SUS): uma avaliação do acesso na Estratégia de Saúde da Família. Interface (Botucatu), Botucatu, v. 14, n. 35, p. 797-810, dez. 2010.
Em relação ao contexto político de gestão, este se mostrou favorável à implantação da Rede, muito embora ainda existam obstáculos a serem superados, no sentido de atender fundamentalmente às necessidades da população, dado que ela é a essência e a razão de ser da RAS.

Nesse sentido, o presente estudo possibilitou constatar a necessidade premente de interligar as diferentes ações e serviços de saúde, a fim de garantir a equidade e a integralidade da atenção, contribuindo para o aperfeiçoamento e reorganização da RAS e subsidiando a tomada de decisão pelos gestores.
BRASIL. Ministério da Saúde. Documento de diretrizes para o cuidado das pessoas com doenças crônicas nas Redes de Atenção à Saúde e nas linhas de cuidado prioritárias. Brasília, DF, 2012.

Ministério da Saúde. Implantação das Redes de Atenção à Saúde e outras estratégias da SAS. Brasília, DF, 2014b.

Ministério da Saúde. Portaria $n^{\circ} 252 / G M$ de 19 de fevereiro de 2013. Institui a Rede de Atenção à Saúde das Pessoas com Doenças Crônicas no âmbito do Sistema Único de Saúde (SUS). Diário Oficial [da] União. Brasília, DF, 19 fev. 2013. Disponível em: $<$ http://bvsms.saude.gov.br/bvs/saudelegis/gm/2013/ prt0252_19_02_2013.html >. Acesso em: 15 out. 2015.

Ministério da Saúde. Portaria $n^{\circ} 483$, de $1^{\circ}$ de abril de 2014. Redefine a Rede de Atenção à Saúde das Pessoas com Doenças Crônicas no âmbito do Sistema Único de Saúde (SUS) e estabelece diretrizes para a organização das suas linhas de cuidado. Diário Oficial [da] União. Brasília, DF, 1 abr. 2014a. Disponível em: $<$ http://bvsms.saude.gov.br/bvs/saudelegis/gm/2014/ prt0483_01_04_2014.html >. Acesso em: 15 out. 2015. 
Ministério da Saúde. Portaria n ${ }^{\circ}$ 4.279, de 30 de dezembro de 2010. Estabelece diretrizes para a organização da Rede de Atenção à Saúde no âmbito do Sistema Único de Saúde (SUS). Diário Oficial [da] União. Brasília, DF, 30 dez. 2010. Disponível em: http://bvsms.saude.gov.br/ bvs/saudelegis/gm/2010/prt4279_30_12_2010.html>. Acesso em: 15 out. 2015.

CHAMPANGE, F. et al. A análise de implantação. In: BROUSSELLE, A. (Org.). Avaliação: conceitos e métodos. Rio de Janeiro: Fiocruz, 2011b.

DENIS, J.; CHAMPAGNE, F. Análise de implantação. In: HARTZ, Z. M. A. Avaliação em saúde: dos modelos conceituais à prática na análise de implantação de programas. Rio de Janeiro, Editora Fiocruz, 1997.

ESCOREL, S. et al. O programa de Saúde da Família e a construção de um novo modelo para atenção básica no Brasil. Revista Panamericana de Salud Pública, Washington, DC, v. 21, n. 2/3, p. 164-176, fev./mar. 2007.

FAUSTO, M. C. R. et al. A posição da Estratégia Saúde da Família na rede de atenção à saúde na perspectiva das equipes e usuários participantes do PMAQ-AB. Saúde em Debate, Rio de Janeiro, v. 38, n. spe, p. 13-33, out. 2014.

GIOVANELLA, L.; ESCOREL, S.; MENDONÇA, M. H. M. Porta de entrada pela atenção básica? Integração do PSF à rede de serviços de saúde. Saúde em Debate, Rio de Janeiro, v. 27, n. 65, p. 278-89, set./dez. 2003.

\section{HARTZ, Z. M. A.; CONTANDRIOPOULOS, A.}

Integralidade da atenção e integração de serviços de saúde: desafios para avaliar a implantação de um "sistema sem muros". Cadernos de Saúde Pública, Rio de Janeiro, v. 20, supl. 2, p. 331-336, 2004.

\section{MARIN, M. J. S.; MARCHIOLI, M.; MORACVICK,} M. Y. A. D. Fortalezas e fragilidades do atendimento nas unidades de saúde tradicionais e da estratégia de saúde da família pela ótica dos usuários. Texto Contexto Enfermagem, Florianópolis, v. 22, n. 3, p. 780-788, jul./ set. 2013

MCLAUGHLIN, J. A.; JORDAN, G. B. Logic models: a tool for telling your program's performance story.
Evaluation and Program Planning, New York, n. 22, p. 65-72, 1999 .

MEDINA, M. G. O contexto local, a organização da atenção primária e a implementação de redes integradas de atenção à saúde: resultados da avaliação de dois estudos de caso. 2006. 269. Tese (Doutorado em Saúde Coletiva) - Instituto de Saúde Coletiva, Universidade Federal da Bahia, Salvador, 2006.

MENDES, E. V. O cuidado das condições crônicas na atenção primária à saúde: o imperativo da consolidação da estratégia da saúde da família. Brasília, DF: OPAS, 2012.

MURRAY, C. J. L.; LOPEZ, A. D. Global health statistics: a compendium of incidence, prevalence and mortality estimates for over 200 conditions. Genebra: WHO, 1996.

OLIVEIRA, R. C. et al. A reorganização da atenção secundária como estratégia para a garantia da integralidade. In: MAGALHÃES JR., H. M. (Org.). Desafios e inovações na gestão do SUS em Belo Horizonte: a experiência de 2003 a 2008. Belo Horizonte: Mazza, 2010.

SANTOS, R. S. A. F. Avaliação da implantação da Rede de Atenção à Saúde das pessoas com doenças crônicas não transmissiveis em um distrito sanitário do município de Recife - Pernambuco. 2015. 205 f. Dissertação (Mestrado em Saúde Pública) - Centro de Pesquisas Aggeu Magalhães, Fundação Oswaldo Cruz, Recife, 2015.

SCHRAMM, J. M.; OLIVEIRA, A. F.; LEITE, I. C. Transição epidemiológica e o estudo de carga de doenças no Brasil. Ciência \& Saúde Coletiva, Rio de Janeiro, v. 9, n. 4, p. 897-908, 2004.

SCHMIDT, M. I. et al. Doenças crônicas não transmissíveis no Brasil: carga e desafios atuais. Lancet, London, v. 377, n. 9781, p. 61-74, maio 2011.

SERAPIONI, M.; SILVA, M. G. C. Avaliação da qualidade do Programa Saúde da Família em municípios do Ceará. Uma abordagem multidimensional. Ciência \& Saúde Coletiva, Rio de Janeiro, v. 16, n. 11, p. 4315-4326, nov. 2011. 
SILVA, D. C. L. Análise da oferta de atenção especializada em uma unidade de referência para portadores de hipertensão e diabetes. 2012. Monografia (Residência Multiprofissional em Saúde Coletiva) - Centro de Pesquisas Aggeu Magalhães, Fundação Oswaldo Cruz, Recife, 2012

SILVA, S. F.; MAGALHÃES, H. M. Redes de atenção à saúde: importância e conceitos. In: SILVA, S. (Org.). Redes de Atenção à Saúde no SUS: o pacto pela saúde e redes regionalizadas de ações e serviços de saúde. 2 ed. Campinas: Saberes Editora, 2011.

SISSON, M. C. et al. Estratégia de Saúde da Família em Florianópolis: integração, coordenação e posição na rede assistencial. Saúde e Sociedade, São Paulo, v. 20, n. 4, p. 991-1004, 2011.

\section{SOCIEDADE BRASILEIRA DE DIABETES. Atlas} do diabetes 2014 - atualização. International Diabetes Federation, 6. ed. Adaptado SBD. Disponível em: $<\mathrm{http} / /$ www.diamundialdodiabetes.org.br/atlas-idf/>. Acesso em: 25 jan. 2015.

SOUZA, E. C. F. et al. Acesso e acolhimento na atenção básica: uma análise da percepção dos usuários e profissionais de saúde. Cadernos de Saúde Pública, Rio de Janeiro, v. 24, supl. 1, p. 100-110, 2008.
SPEDO, S. M.; PINTO, N. R. S.; TANAKA, O. Y. O difícil acesso a serviços de média complexidade do SUS: o caso da cidade de São Paulo, Brasil. Physis, Rio de Janeiro, v. 20, n. 3, p. 953-972, 2010.

TANAKA, O. Y. Avaliação da atenção básica em saúde: uma nova proposta. Saúde e Sociedade, São Paulo, v. 20, n. 4, p. 927-934, out./dez. 2011.

TOBAR, F.; YALOUR, M. R. Como fazer teses em saúde pública: conselhos e idéias para formular projetos e redigir teses e informes de pesquisas. Rio de Janeiro: Editora Fiocruz, 2001.

VIEIRA, F. S. Avanços e desafios do planejamento no Sistema Único de Saúde. Ciência e Saúde Coletiva, Rio de Janeiro, v. 14, supl. 1, p. 1565-1577, out. 2009.

\footnotetext{
Recebido para publicação em maio de 2015

Versão final em outubro de 2015

Conflito de interesses: inexistente

Suporte financeiro: Fundação de Amparo à Ciência e Tecnologia de Pernambuco (Facepe), número do processo: APQ-02774.06/13; Conselho Nacional de Desenvolvimento Científico e Tecnológico (CNPq), Número do processo: 563911/2010-7
} 